\title{
El discurso y su influencia en los medios. Análisis de resultados del discurso ambientalista ${ }^{1}$
}

\author{
The discourse and its influence in the media. Analysis of environmental \\ discourse results
}

\author{
Metzli Sofía, Jiménez Tovar ${ }^{2}$ \\ Universidad La Salle, México \\ https://orcid.org/0000-0001-9640-6221 \\ https://doi.org/10.29105/pgc7.13-3
}

\begin{abstract}
RESUMEN
El presente artículo es producto de una investigación que tuvo por objetivo proponer el uso del análisis de discurso y sus resultados, como una forma de demostrar el efecto que tienen los discursos sobre los medios de comunicación, y por ende sus consecuencias en la sociedad, así como el impacto de los líderes de opinión. Se aplicó el método hermenéutico, con las técnicas de investigación análisis de discurso y monitoreo de medios, el tipo de investigación es mixta. Se concluyó que la cobertura mediática fue variable, y que la interpretación de los discursos sí puede brindar un panorama contextual de la realidad y los cambios que se generaron con el tiempo; que los discursos al final cumplen con su objetivo, que es generar accion y obtener una mayor cobertura mediática al convertir el cambio climático en un tema de interés público.
\end{abstract}

Palabras claves: Análisis de discurso, análisis de resultados, cambio climático, prensa

\section{ABSTRACT}

This article is the product of research aimed at proposing the use of speech analysis and its results, as a way of demonstrating the effect of speeches on the media, and thus their consequences on society, as well as the impact of opinion leaders. The hermeneutic method was applied, with the techniques of discourse analysis research and media monitoring, the type of research is mixed. It was concluded that media coverage was variable, and that the interpretation of speeches can provide a contextual picture of reality and the changes that were generated over time; that speeches in the end meet their goal, which is to generate action and gain greater media coverage by turning climate change into a public interest issue. Keywords: Climate change, discourse analysis, newspapers results analysis.

Recibido: 11 de Abril 2020 - Aceptado: 15 de Junio 2020

Cómo referenciar este artículo:

Jiménez Tovar, M. S. (2021). El discurso y su influencia en los medios. Análisis de resultados del discurso ambientalista. Revista Politica Globalidad y Ciudadanía, 66-92. Recuperado de http:// revpoliticas.uanl.mx/index.php/RPGyC/article/view/167. https://doi.org/10.29105/pgc7.13-3

\footnotetext{
1 Este artículo es producto del proyecto terminal "El poder de la palabra. Análisis de resultados con base en la hermenéutica del discurso medioambiental”. Iniciado en 2019 y finalizado en 2020.

2 Licenciada en Ciencias de la comunicación por la Universidad de la Salle.Email: mj@lasallistas.org.
} 


\section{1.- INTRODUCCION}

El cambio climático es un problema que ha tomado relevancia en los últimos años en diferentes ámbitos, requiriendo con mayor urgencia soluciones que contrarresten las letales consecuencias que atentan contra cualquier forma de vida en el planeta. Por ende, al examinar información disponible sobre el tema, en específico de las múltiples dependencias de la ONU enfocadas en el medio ambiente, la premisa principal que permea es la "acción inmediata". Una propuesta para lograr este objetivo es por medio del discurso, debido a su carácter generador de acción (Van Djik, 2012) porque "la palabra, aunque no sirve para representar ni para transmitir la realidad, es útil para persuadir a los hombres" (Hernández y García, 1994). Al unir una manera de comunicación de gran alcance como es el discurso, y la prensa, alias periódico, que es el medio de comunicación más longevo con un alcance igual de amplio y con trascendencia efectista, se podrían obtener resultados de gran impacto.

Por ello el objetivo principal de este trabajo es conocer el alcance que tienen los discursos medioambientales enfocados en el cambio climático sobre los contenidos que se publican en diversos periódicos en el continente americano, respondiendo a la pregunta de investigación ¿se logró una mayor cobertura mediática acerca del cambio climático en el periodo entre el discurso de José Mujíca en la Conferencia de las Naciones Unidas sobre el Desarrollo Sostenible en 2012 y el discurso viral emitido por Greta Thunberg en el Parlamento Europeo en 2019?

Con base en ello se determinó la influencia que los discursos, y en consecuencia los líderes de opinión tienen sobre la prensa; así como proponer un enfoque distinto de la metodología mixta que se empleará con el análisis de discurso y el mapeo de medios, los cuales aporten la información necesaria para realizar un análisis de resultados respecto a soluciones comunicativas que funjan como respuesta a problemáticas sociales.

Una limitante es el carácter diacrónico de la investigación, ya que el período que se contempló para la recolección de notas informativas fue de 7 años, por lo que 
la información disponible puede tener variables importantes que permeen los resultados debido al número impreciso de noticias que se han publicado a lo largo de ese plazo. Otra variable a considerar es el mapeo de medios, por su carácter cuantitativo omite aspectos cualitativos en el contenido de las notas informativas como la ideología del periódico y del periodista, las cuales llegan a transmitir diversas posturas sobre el tema, por ende no se genera una opinión unificada en la población; al igual este análisis despoja de individualidad a los emisores relegando su libre albedrío ya que "cada individuo o grupo social concibe de manera diferenciada el problema, esto en función de sus experiencias, información, conocimiento y contexto" (Arias y Rosales, 2019, p. 248).

El interés principal de este trabajo es estudiar la creciente y compleja exposición de medios de comunicación a la que estamos sometidos los seres humanos como lo muestra IAB México (2017) en su estudio de consumo de medios. Los medios son constructores de la realidad, por ello, la información que se publica en el medio cobra relevancia en la vida de las personas, si se logra medir la cantidad de información transmitida se puede tener una aproximación de la posición que ocupan ciertos temas en la sociedad, esto se puede lograr examinando los siguientes factores: la proporción de noticias sobre la problemática; desde que temas o perspectivas se aborda: el lugar geográfico del periódico y el nombre del periódico en que se publican; los líderes de opinión o personalidades que se vinculen con el tema; el tipo de documento periodístico; y el idioma en que está presentado; estas variables fueron definidas por las categorías de información encontradas en la base de datos ProQuest, que fungió como instrumento del mapeo de medios. De acuerdo con Van Djik (2012) se puede medir la evolución de un acontecimiento social conforme el lenguaje empleado en los discursos, esta premisa fungió de base para el análisis de resultados.

En general, los artículos o estudios disponibles respecto al análisis de discurso tienen como objetivo entender y ampliar el conocimiento respecto a la problemática social abordada, algunos estudios han intentado emplearlo como un análisis de resultados social, sin embargo, no se encuentran caracterizados como tal. Por ello 
el alcance de este análisis es global, debido a diversos factores; el primero es la elección de los líderes, ya que apelan a diversas realidades, contextos y culturas, por un lado, se encuentra el exmandatario José Mujíca de Uruguay, y por otro está Greta Thunberg la joven activista sueca; y el segundo factor que amplia su alcance es el mapeo de medios, que abarca desde prensa con un efecto mundial hasta prensa de localidades específicas.

\section{2.- FUNDAMENTO TEÓRICO}

Para poder entender y explicar el efecto del cambio climático, así como sus causas, se deben exponer ciertos conceptos básicos. De acuerdo con la Organización de las Naciones Unidas (ONU) en el módulo 1 de su diplomado "Introducción a la Ciencia del Cambio Climático", (ONU, s.f.) se muestra que hay una diferencia entre clima y tiempo, los dos fenómenos ocurren en la atmósfera pero, si se suscita en un momento determinado es "tiempo", este varía en un espacio temporal corto: en días, semanas, etc. que incluye la temperatura, la velocidad del viento, la humedad, la presión barométrica, entre otros componentes; por otro lado el "clima" es el promedio del estado del tiempo, que son períodos prolongados.

La ONU (s.f.) expone que el clima es el estado del sistema climático que se compone por: la atmósfera, que es la envoltura gaseosa que rodea a la Tierra; la hidrósfera, parte de la Tierra que involucra las aguas en estado líquido tanto las superficiales como las subterráneas como los océanos, ríos, lagos, etc.; la criósfera, que es la capa que conforma el agua en estado sólido; superficie terrestre, es la capa más externa de la superficie sólida de la Tierra y de los océanos, que es donde se produce la actividad volcánica e influye en el clima; y la biósfera, que comprende a todos los organismos vivos así como los ecosistemas de la superficie terrestre y océanos. Todos estos elementos determinan e influyen en el estado y la dinámica del clima terrestre. Uno de los mecanismos más importantes del sistema climático es el efecto invernadero, este se genera a partir del desequilibrio entre la energía que recibe la Tierra por parte del Sol y la energía que se libera al espacio exterior, ya que es cada vez menor la cantidad de energía calórica que logra irradiarse al espacio por lo que se queda en la atmósfera a causa de los Gases de Efecto 
Invernadero (GEI), los cuales se producen por un exceso de dióxido de carbono (CO2), esto aumenta la temperatura de la Tierra causando estragos irreversibles hacia cualquier forma de vida en el Planeta.

El calentamiento global es un cambio climático que se ha investigado desde inicios del siglo XX por ser un variación en el clima sin influencias naturales, que ha ocurrido a una velocidad nunca antes vista en otro fenómeno climático registrado. Ya que su principal causa es el aumento de concentración de gases de efecto invernadero en la atmósfera, estos se han producido desde la Revolución Industrial a finales del siglo XVIII, por estos componentes que absorben y emiten radiación térmica, se tiene una mayor cantidad de calor en la atmósfera que incrementa la temperatura global, las repercusiones que tiene este efecto se le denomina "Cambio Climático Antropogénico" que son las acciones provocadas por el hombre. (Oceana, s.f.)

De acuerdo con Velázquez de Castro (Agosto 2012) son cuatros rasgos los que caracterizan la crisis ambiental: la globalidad de problemas ambientales que afectan a toda la Tierra sin haber sido generados por el planeta, sino por una pequeña parte de la población; rapidez o exponencialidad, a lo largo de la historia humana han existido múltiples cambios climáticos sin embargo ningún ha sido tan fulminante; el aumento de problemas ambientales que al final solo permite la acumulación de sustancias en los organismos; y la persistencia, los prolongados efectos secundarios de los impactos generados, ya que algunos pueden permanecer más de un siglo en el planeta.

Por ello se requieren realizar acciones que ayuden a contrarrestar estos efectos, que informen la situación, y el discurso es uno de los elementos que puede aportar a esta causa porque como lo expone el lingüista Van Djik (1978) “a través del control de la mente se pueden controlar los actos, se tiene un poder increíble sobre la sociedad porque la gente va a hacer o no hacer lo que uno quiere que haga".

Los discursos realizan el "arte de persuadir por medio de la palabra" (Dahdah-Antar, 2019, p. 112) se busca impulsar un cambio, para ello es importante conocer 
el funcionamiento y estructura de los discursos argumentativos. El filósofo Paul Ricoeur (1995) expone que el discurso es una expresión del lenguaje conformada por palabras, las cuales separadas entre sí no conforman nada, no tiene intención alguna, es la oración la que llega a significar algo. La oración es un conjunto de palabras, por ello, el discurso necesita un verbo y un nombre, según el autor, las cuales cuentan con una conexión que trasciende las palabras. Esta definición dota al receptor o interprete con una responsabilidad, y un poder, de darle un significado a las palabras, adaptarlas a su realidad, por lo que al momento de generar un análisis se debe tomar en cuenta no solo el lenguaje si no el contexto y la cultura en la que se elaboró el texto del discurso, así como el contexto en el que se emite. Pensamiento que respalda el lingüista Van Djik (2012) al exponer que las situaciones sociales, políticas y culturales influyen en la interpretación subjetiva, y afirmando que las situaciones sociales son constructos sociales que pueden influir en toda la conducta humana.

Otro de los grandes exponentes del análisis de discurso es Foucault (1987), quien muestra y propone normas que rigen los discursos: el qué se dice, cómo y en qué circunstancia, que responden a tres motivos el poder, el peligro y la reducción de incertidumbre.

Al entender los aspectos que definen y engloban al discurso la pregunta que surge es ¿con base en qué aspectos se puede generar un análisis del discurso? Por lo que Philips y Hardy (2002) presentan un esquema que expone las diversas vertientes a las que se puede dirigir un análisis de discurso:

Categoriza las opciones de acuerdo con dos dimensiones clave: el nivel en el que se encuentra el enfoque del discurso ya sea en textos individuales o en el contexto que los rodea; el otro nivel es en el que se enfoca la investigación, si es en poder o ideología al contrario de la construcción social. De acuerdo con las 4 propuestas de análisis: análisis lingüístico social; estructuralismo interpretativo; análisis crítico del discurso; y análisis lingüístico del discurso. La mayoría tiene un interés sobre las relaciones de poder en los discursos, por ello la categoría que se adecua mejor al enfoque de la investigación es el análisis lingüístico social. 
Este tipo de análisis es constructivo y se basa en los textos. Los investigadores se enfocan en textos individuales relacionándolos marginalmente al contexto distante en el que se generaron o se exploran las dinámicas de poder en las que están implicados. Los estudios de este tipo se enfocan en los aspectos constructivos del texto, ayudan a entender no solo las micro dinámicas del discurso de decisiones individuales, sino que también ayuda en la comprensión de los fundamentos de la realidad social del discurso en la que se encuentran las relaciones.

Al identificar el objetivo y enfoque del análisis de discurso, la aportación de la hermenéutica es con base en su carácter epistémico, el cual se entiende como "el estudio de la naturaleza del conocimiento y la justificación, en específico el estudio de sus características definitorias, sus condiciones sustantivas; y los limites del conocimiento y la justificación" (Audi, 2004)

Al final el objetivo del epistemólogo es el de proveer de componentes definitorios del conocimiento, que favorezcan al análisis del conocimiento. El análisis estándar se compone por tres elementos: la justificación, la verdad, y la opinión o creencia. La justificación epistémica se limita a la aportación de evidencias, a aumentar la verdad y reducir la falsedad.

Al entender las aportaciones y relevancia de las metodologías, se complementa con una teoría social, que refuerza la importancia de encausar los contenidos hacia un bien común por el poder mediático efectista. Esto por medio de una teoría que, para muchos pensadores, puede ser obsoleta sin embargo al observar nuestro entorno nos podemos dar cuenta que sigue en vigor, la Teoría de la Comunicación en dos pasos, para Paul Lazarsfeld et al. (1944), sociólogo austríaco, fundador del análisis sociológico norteamericano quien basó sus estudios en los efectos que causan los medios de comunicación los cuales en ocasiones alienan a la población, por medio de la teoría de la Comunicación de masas (Lazarsfeld, 2012), y una ramificación de esta teoría son los líderes de opinión creados por los medios, y deben tener características determinadas para que los receptores logren empatizar con los ideales o ideas que venden. El flujo de la comunicación en la teoría de dos pasos es que la información primero llega a los líderes de opinión 
quienes influyen y la transmiten a las personas con las que se encuentran en contacto mediático. Los medios no cambian la forma de pensar sólo la refuerzan, esto lo hacen a través de líderes de opinión que son personas que se localizan en diferentes estratos sociales y ocupaciones, deben fungir como un modelo para otros e influenciar (Lazarsfeld, 2012, p. 142). Sin embargo, en este trabajo, se realiza una nueva propuesta de enfoque a la teoría, se plantea que ya no son los medios la única fuente de la información, al contrario, la iniciativa es que los hechos noticiosos ahora son dictaminados por los líderes de opinión, ellos son quienes manejan los contenidos, establecen qué, quién, cuándo y de qué se hablará. Con base en esto se seleccionó a las figuras públicas que sirvieron como objeto de estudio, Greta Thunberg y José Mujíca.

Dentro de las diversas alternativas de medios de comunicación, se seleccionó la prensa por la estrecha relación que desde su nacimiento ha tenido con el poder, en 1850 el periodista F. Knight Hunt nombró su libro "El Cuarto Estado" dotando al periódico de un nuevo objetivo y término, el cual surgió a partir de la división de tres poderes gobernantes: ejecutivo, legislativo y judicial; siendo la prensa la que funja como un órgano fiscalizador del gobierno, ya que en general la prensa se encuentra privatizada, lo cual otorga cierta autonomía y desapego del Estado, independencia que le permite ser crítica de los gobernantes o acontecimientos que lo ameriten.

La relevancia de este medio radica en el poder que tiene para expandir el conocimiento, en los primeros años posteriores a su concepción los empresarios lo percibieron como una ventana de oportunidad como publicidad o propaganda, siendo en la actualidad uno de sus principales ingresos. De igual forma al comienzo comentaban que los periódicos estimulaban en los lectores el escepticismo, ya que propició a que los lectores se empezarán a cuestionar la información porque les brindaba diferentes perspectivas de los acontecimientos, sin embargo, el aporte más grande que se le atribuye fue crear la opinión pública. 


\section{3.- METODO}

\section{Diseño}

El discurso es un indicio de la realidad social, de los pensamientos, no es un espejo o reflejo de la realidad, por lo que se seleccionó el análisis de discurso como metodología puesto que su relevancia recae en el poder de analizar el discurso como una forma de acción social, encausando el análisis en el contenido de las notas informativas para poder conocer el aporte que han tenido los discursos en el medio tradicional, la prensa.

En primera instancia, se realizó la selección de los líderes de opinión y de los discursos con base en el aporte, popularidad, contexto y relevancia para, de esta manera, poder contrastar aquellos aspectos que coincidan, así como entender las diferencias particulares.

En el análisis de discurso se empleo como base el modelo empleado en el artículo del sociólogo Alejandro Rico (2004) el cual realizó una caracterización de los elementos del discurso como: unidades temáticas, estructuras argumentativas, tópicas y marco teórico. Basándose en el estudio de la estructura interna del discurso. Para este trabajo se preseleccionaron unidades discursivas de análisis las cuales surgieron de los conceptos que involucran una problemática social y cambio climático, a su vez se agregaron conceptos puntuales de cada uno de los líderes seleccionados antes mencionados.

Las unidades que se emplearon se dividieron en categorías y subcategorías, las categorías que de acuerdo con el análisis realizado permearon el discurso de $\mathrm{Mu}-$ jíca fueron: Economía, ecología, humanidad y tiempo. Por su parte las categorías de la joven activista Thunberg fueron: Política, medio ambiente y sociedad.

Las subcategorías las conforman conceptos que deriven o se vinculen con las categorías principales tales como: desarrollo, sociedad, mercado, recursos naturales, entre otros.

Para el monitoreo de medios se basó la metodología en un modelo empleado por 
la Doctora en Sociología Maricarmen Cárdenas (2010) quien realizó un estudio sobre el calentamiento global y su tratamiento en los medios, en el cual el seguimiento de medios se realizó por medio de la base mediática diaria de la Secretaría de Medio Ambiente y Recursos Naturales (SEMARNAT), la cual dictaminó las categorías de noticias, y la clasificación general. Con ello está investigación versó sobre la base de datos ProQuest, quien a su vez dictaminó las categorías y clasificaciones de análisis.

Con bases en las unidades de análisis que se encontraron en el discurso, estas se emplearán para dos análisis, el primero será un análisis comparativo entre los dos discursos puesto que por medio de las unidades se medirá la evolución del problema y se pueden analizar los cambios que se hayan suscitado durante los 7 años entre la emisión del discurso de José Mujíca y el discurso de Greta Thunberg. Y en segundo lugar, conforme a un análisis cuantitativo sincrónico de notas informativas se analizará el número de notas generadas y encontradas en la base de datos ProQuest que contengan la palabra "cambio climático" al ser el tema principal de este trabajo, el límite temporal del análisis de medios fue dictaminado por los discursos, en específico por el momento de su comunicación. Con el fin de obtener una contextualización más precisa y oportuna se elaboró una sub selección sincrónica de estas publicaciones noticiosas donde se analizarán a partir del día de la emisión de cada discurso diez meses antes y diez meses después, para tener un panorama lato sensu. La elección de 10 meses fue condicionada por la temporalidad del discurso emitido por Greta, a causa de ser contemporáneo a la investigación no concede llegar a una evaluación de 12 meses.

Por consiguiente, se realizaron tres categorizaciones de las publicaciones en periódicos, la primera abarcó el número de publicaciones que tuvieran la palabra cambio climático y fue desde el 1 de septiembre de 2011 al 20 de junio de 2012 (día que se presentó el discurso de Mujíca) y del 20 de junio de 2012 al 20 de marzo de 2013. El mismo periodo se empleo para el discurso de Greta, diez meses, que corrieron del 1 de julio de 2018 al 17 de abril de 2019, día que se emitió el discurso, y del 17 de abril de 2019 al 31 de enero de 2020 que son 10 meses después. 


\section{Participantes}

Se decidió abrir con el análisis del discurso del ex mandatario José Mujíca por el cargo de poder que logró al provenir de una infancia con bajos recursos y por la ideología que permeó su carrera política, y sigue permeando, en pro del medio ambiente, las personas de escasos recursos y el bienestar común, como lo muestra el diario El País (2018). La elección del discurso se debe a la relevancia que tuvo en la época que se emitió ya que funge como un hito porque no solo expuso la problemática medioambiental, sino que lo hizo por medio de una fuerte crítica al sistema económico y gobernante. Otro aspecto importante de resaltar para el análisis es la Conferencia en la que se propagó el discurso, la cual se dio a conocer con el acrónimo "Río+20". En esta conferencia se creó el documento "El futuro que queremos" de la Organización de las Naciones Unidas (ONU, 2012), acuerdo que firmaron todos los países participantes, y sirvió como base para la creación de la Agenda 2030 y los reconocidos Objetivos de Desarrollo Sostenible (ODS) publicados en 2015 por la ONU.

La inclusión de Greta Thunberg en el análisis es a causa de la apresurada fama e impacto mediático que logró la activista de acuerdo con el medio The Guardian (Belam, 2019), y el aporte que puede brindar al ser un individuo completamente ajeno a José Mujíca como en el nivel de estudio, el entorno social, el nivel de involucramiento que tienen con la política, la edad, etc. y de esta forma contrastar los aportes de cada uno. La selección del discurso, es por razón de que pese a no ser su primera aparición pública ni la más evocada, si fue la ponencia donde se le procedió a percibir con la relevancia y categoría de una líder de opinión, en ese momento se reconoció que no sería un personaje de una sola aparición, si no que se convertiría en una figura recurrente en eventos de gran magnitud efectista.

\section{Instrumentos}

Para el análisis de discurso se empleó el software Atlas.ti por su eficiencia en las investigaciones cualitativas. Dentro del programa se emplearon los recursos: contador de palabras, creación de unidades de investigación y nube de palabras. Estos 
recursos facilitaron la comparación y análisis de los objetos de estudio.

Para el mapeo de medios, se utilizó la base de datos ProQuest por la amplia cobertura de información dentro del World Wide Web, porque su funcionamiento radica en colaboraciones con múltiples organismos, asociaciones, instituciones, entre otros. De igual forma resultó el instrumento idóneo por la facilidad de categorización y filtración dentro de la plataforma, así como el respaldo académico internacional que dispone.

\section{Procedimientos}

Para empezar, se hizo la transcripción de los discursos, en el caso del discurso de Greta al ser emitido en el idioma inglés requirió adicionalmente una correcta traducción. Con base en las transcripciones se obtuvo un conteo general de palabras, después se realizó la concordancia para conocer las unidades básicas que se encontraron en el discurso, y en consecuencia se obtuvo una nube de palabras que ejemplifica las unidades con mayor recurrencia.

Al tener el análisis de los dos discursos, en específico las nubes de palabras y conteo de unidades básicas, se tomó como base las unidades del discurso de José Mujíca y se hizo una comparación con las unidades básicas en el discurso de Greta, se observaron las unidades que se repetían y el número de ocasiones que se encontraron, con ello se refutó la hipótesis de si existía o no una evolución en la problemática social.

Después se empleo el mapeo de medios, que mostró el número de documentos producidos que contienen la palabra "cambio climático" en el periodo seleccionado de cada líder de opinión, y en el periodo entre cada discurso. En el ciclo individual, primero se analizaron los datos del mes en el que se emitió el discurso, se ocupó ese número como base y se vio el crecimiento o decrecimiento de cobertura mediática de los meses delimitados. Por su parte, del periodo intermedio entre cada discurso a parte del análisis de datos de producción y cobertura mediática, también se realizó un análisis con el apoyo de las unidades básicas, ya que con el mapeo de medios existe una división que arrojó las temáticas con respecto al 
cambio climático que se localizaron en los documentos, por lo que al tener los conceptos y sus respectivos datos de repetición, se contrastó con las unidades básicas y se obtuvo la medición de la influencia directa de los discursos sobre el contenido del medio.

\section{4.- RESULTADOS}

En el análisis comparativo de los discursos con base en las unidades de análisis (Figura 1) podemos observar que las unidades de mayor relevancia en el discurso de José Mujíca son: gobierno, sociedad y vida; ya que son las 3 unidades con mayor número de menciones siendo la cantidad máxima 8. En el caso de Greta la unidad con mayor mención fue "destrucción" siendo 18 el número máximo, seguida de "actuar" con 14 repeticiones y "casa" con 12. Las unidades que se repitieron en los dos discursos son:

$\begin{array}{llll}\text { - Acuerdo } & \text { - Economía } & \text { - Negación } & \text {-Sociedad } \\ \text { - Civilización } & \text { - Futuro } & \text { - Pasado } & \text { - Tiempo } \\ \text { - Contaminación } & \text { - Globalización } & \text { - Planeta } & \\ \text { - Destrucción } & \text { - Medio ambiente } & \text { - Política/políticos }\end{array}$

Puesto que el total de códigos en los discursos es de 50 en el caso de Mujíca y 49 en el caso de Greta, el promedio sería 49.5 siendo este nuestro universo, el número de conceptos que intersectan es 14 por lo que el porcentaje es de $28.28 \%$ de conceptos compartidos.

En la Figura 1 podemos observar que las categorías están diferenciadas por color para una mejor identificación, conforme el número de subcategorías que conforman las categorías, podemos observar que la categoría, por consiguiente, el tema, con mayor peso es: "economía" en el discurso de José Mujíca y con Greta Thunberg "medio ambiente".

Con base en estos resultados se puede responder al objetivo específico de verificar si hubo una evolución o mejora, demostrando que no existió una mejora porque 
el tema medioambiental pasó de un tercer a un primer plano; otros datos que respaldan esta conclusión son las unidades con mayor número de mención a causa de que en el 2012 con Mujíca los temas más relevantes estaban relacionados a las sociedades, mientras que las unidades en el 2019 con Greta develan el deterioro puesto que la unidad encontrada más veces fue "destrucción" seguida de "actuar", haciendo alusión a la urgencia de realizar un cambio.

Respecto al mapeo de medios, realizado con base en la información disponible en la base de datos ProQuest, de los 3 filtros temporales aplicados son:

Filtro 1, los 20 meses del discurso de José Mujíca se dividieron en dos:

- 10 meses anteriores al discurso (Filtro 1.1)

- 10 meses posteriores, empezando el 21 de junio de 2012 (Filtro 1.2);

Filtro 2, 20 meses del discurso de Greta Thunberg divididos en dos:

- 10 meses anteriores al discurso empezando el 1 de julio de 2018 al 17 de abril de 2019 (Filtro 2.1)

- $\quad$ los 10 meses posteriores, empezando el 18 de abril de 2019. (Filtro 2.2)

Filtro 3, el periodo de latencia que abarca del 21 de junio de 2012 hasta el 17 de abril de 2019

El total de publicaciones en periódicos que se obtuvieron del Filtro 1.1 (Gráfico 1) fue de 1,290 publicaciones, siendo junio el mes con menor número de publicaciones 90, mes que se emitió el discurso y se llevo a cabo la Conferencia Río+20, y el mes que mostró la cantidad máxima fue diciembre de 2011 con 161 . Respecto al Filtro 1.2, el total de notas publicadas en el periodo fue un total de 1,083, siendo el mes de junio el que presentó el menos número, como se muestra en el Gráfico 2, con 53 publicaciones, es importante recalcar que a comparación del Filtro 1.1, las 53 publicaciones respectan a los últimos 10 días del mes por lo que se presentó un incremento del $17.7 \%$ en la producción y publicación de documentos de ese mes, de acuerdo con la gráfica en los meses a posteriori hubo un incremento gradual 
de noticias. Sin embargo, al comparar el total se muestra que la producción fue superior antes del discurso que después.

Los resultados del Filtro 2.1 (Gráfico 3) arrojan que el número de publicaciones en ese periodo fue de 827 notas publicadas siendo los primeros 17 días de abril el número más bajo de publicaciones con una cantidad de 48 , y febrero el más alto con 112. En el Gráfico 4 (Filtro 2.2) el total de publicaciones fue de 1,152, siendo los 13 días restantes de abril el mes con una menor cantidad, 41; y junio mostró el número máximo con 155 documentos. De acuerdo con los resultados, posterior al discurso, durante el mes de abril hubo un incremento del 11\% de notas publicadas respecto al cambio climático. Y como se puede observar en los Gráficos 3 y 4 si existe una mayor producción de documentos a posteriori a la propagación del discurso ya que la cantidad a priori es de 827 menos a la cantidad a posteriori de 1,152 , lo cual se refleja en un incremento de casi $40 \%$.

De acuerdo con el Filtro 3 (Gráfico 5) se puede observar un aumento gradual en la publicación de documentos, siendo el 2015 el año con mayor producción, sin embargo, de la misma manera la gráfica tendió a decrecer los años posteriores, hasta el 2019. Al completar la información de la Gráfica 5 con las Gráficas 1 y 4 , decantando los datos a solo los años en los que se propagaron los discursos que son 2012 y 2019, en la primera mitad del 2012 (del 1 de enero al 20 de junio) el número de notas publicadas fue de 739 y la última mitad (del 21 de junio al 31 de diciembre) de 637, lo cual muestra que no hubo un incremento de producción de noticias con contenido del cambio climático, puesto que fue de $0.44 \%$. En el caso de Greta Thunberg las notas publicadas del 1 de enero al 17 de abril de 2019 fueron 325, y los meses restantes desde el 18 de abril de 2019 al 31 de diciembre de 2012 fueron 1,006, lo cual demostró un crecimiento de apenas $0.26 \%$, menor al porcentaje de José Mujíca. Sin embargo, al aumentar el periodo de análisis hasta el 31 de enero se muestra un incremento en $11 \%$.

Estos resultados confirman parcialmente la hipótesis, a causa de que, si existió un incremento durante los primeros 4 años posteriores al discurso de Mujíca, sin embargo, decreció gradualmente por que el efecto que tuvieron los discursos en 
los medios fue limitado y condicionado, puesto que no fueron los únicos líderes de opinión o figuras públicas que abordaron el tema.

El último análisis combina las unidades básicas de investigación (Figura 1) con los temas encontrados en las notas periodísticas del período 21 de junio de 2012 a 17 de abril de 2019 (Tabla 1), se ocupan solo las unidades con mayor número de repetición del discurso de José Mujíca, debido a que la periodicidad del filtro de los temas no incluye tiempo posterior al discurso de Greta, en los cuales se encontró que uno de los temas más encontrados es presidentes, similar a la unidad del discurso "gobierno" y "autoridades". En temas se encontraron conceptos con un amplio número de repeticiones: partidos políticos, crecimiento económico, producto interno bruto (PIB) e impuestos, los cuales hacen referencia a la categoría con mayor relevancia de Mujíca la "economía” por lo que si existió un porcentaje de influencia en los periódicos.

Figura 1: Unidades básicas obtenidas del análisis de discurso

\begin{tabular}{|c|c|c|c|c|c|c|}
\hline & 0 & Nombre & ๑ & 0 & Nombre & () \\
\hline$\diamond$ & $\bullet$ & acompaగar & - & $\diamond \bullet$ & politicos & $\longrightarrow 16$ \\
\hline$\diamond$ & $\bullet$ & acuerdos & - & $\diamond \bullet$ & sociedad & $\Longrightarrow 7$ \\
\hline$\diamond$ & - & animales & 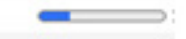 & $\diamond 0$ & actuar & $\longrightarrow 14$ \\
\hline$\diamond$ & $\bullet$ & autoridades & $\longrightarrow$ & $\diamond \bullet$ & datos duros & $\Longrightarrow \epsilon$ \\
\hline$\diamond$ & $\bullet$ & causa & 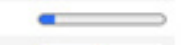 & $\diamond 0$ & necesario & $\Longrightarrow \epsilon$ \\
\hline$\diamond$ & - & civilización & $\longrightarrow$ & $\diamond \bullet$ & consecuencia & $\longleftrightarrow 1$ \\
\hline$\diamond$ & $\bullet$ & competencia & 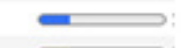 & $\diamond \bullet$ & soluciones & $\longrightarrow \mathrm{g}$ \\
\hline$\diamond$ & $\bullet$ & consecuencias & 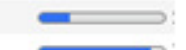 & $\diamond$ & calentamiento global & $\Longrightarrow 6$ \\
\hline$\diamond$ & $\bullet$ & consumo & $\longrightarrow$ & $\diamond \bullet$ & perjudicial & $\Longrightarrow \epsilon$ \\
\hline$\diamond$ & $\bullet$ & contaminación & 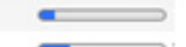 & $\Leftrightarrow$ & acuerdos & $\Longrightarrow 2$ \\
\hline$\diamond$ & $\bullet$ & crisis & - & $\diamond 0$ & negación & $\Longrightarrow \mathrm{E}$ \\
\hline$\diamond$ & $\bullet$ & cultura & $\longrightarrow:$ & $\diamond \bullet$ & responsabilidad & $\Longrightarrow 3$ \\
\hline$\diamond$ & $\bullet$ & desarrollio & $\longrightarrow$ & $\diamond \bullet$ & anticipado & $\Longrightarrow 2$ \\
\hline$\diamond$ & $\bullet$ & desechable & $=$ & $\diamond$ & desinformación & $\Longrightarrow 2$ \\
\hline$\diamond$ & $\bullet$ & desperdicio & $\square$ & $\diamond \bullet$ & destrucción & $\longrightarrow$ \\
\hline 8 & • & $\begin{array}{l}\text { destrucción } \\
\text { discusión }\end{array}$ & $\longrightarrow$ & $\diamond \bullet$ & cambios & $\longrightarrow 8$ \\
\hline$\Leftrightarrow$ & $\bullet$ & ecología & $\Longrightarrow$ & $\diamond \bullet$ & combustibles tósiles & $\longrightarrow 7$ \\
\hline 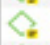 & $\bullet$ & economía & י & $\diamond \bullet$ & tiempo & $\Longrightarrow \mathrm{E}$ \\
\hline 8 & $\bullet$ & felicidad & $\longrightarrow$. & $\diamond \bullet$ & redueción & $=4$ \\
\hline$\diamond$ & $\bullet$ & futuro & $\Longrightarrow$ & $\diamond \bullet$ & voto & $=4$ \\
\hline$\diamond$ & $\bullet$ & globalización & $\longrightarrow:$ & $\diamond \bullet$ & elima & $\Longleftrightarrow 2$ \\
\hline$\Delta$ & $\bullet$ & gobierno & : & $\diamond \bullet$ & organizaciones & $\Longrightarrow 2$ \\
\hline$\diamond$ & $\bullet$ & humanidad & $\longrightarrow$ : & $\diamond \bullet$ & planeta & $\longrightarrow 8$ \\
\hline$\diamond$ & $\bullet$ & vida & $\longrightarrow$ & $\diamond \bullet$ & futuro & $\longrightarrow$; \\
\hline \multirow[t]{4}{*}{$\diamond$} & - & voluntad & 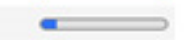 & $\diamond \bullet$ & pánico & $=5$ \\
\hline & & & & $\diamond \bullet$ & medios & $=4$ \\
\hline & & & & $\diamond \bullet$ & nif̂os & $=4$ \\
\hline & & & & $\diamond \bullet$ & celebrar & $=3$ \\
\hline
\end{tabular}


Fuente: Elaboración propia en Atlas.ti (2020). Nota: Con base en las unidades se realizó el análisis comparativo de los discursos, así como las nubes de palabras. En el costado derecho muestra el número de veces encontradas en el discurso.

Figura 2: Nube de palabras del discurso de José Mujíca

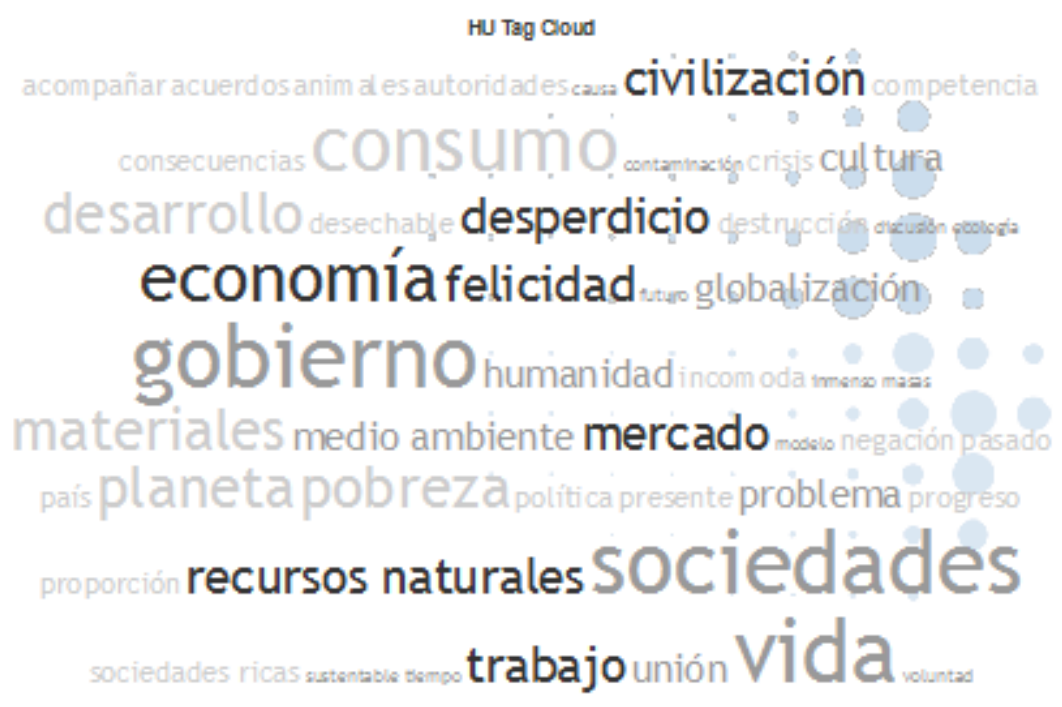

Fuente: Elaboración propia en Atlas.ti (2020).

En esta figura se ilustran las unidades de análisis encontradas en el discurso, siendo las palabras con mayor jerarquía visual aquellas que cuentan con una mayor recurrencia, definidas por la saturación de color y el tamaño de letra.

Figura 3: Nube de palabras del discurso de Greta Thunberg.

HU Tag Cloud

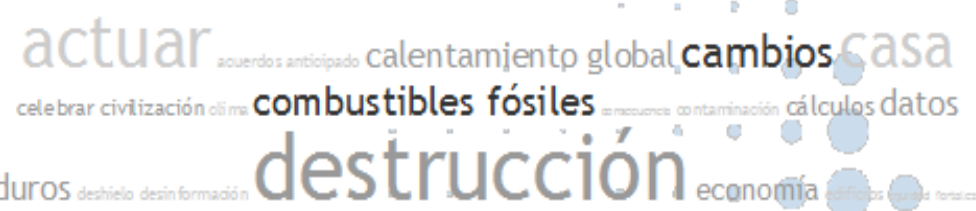

futuro global masteinsertiduntere juticia me dio ambiente medios necesarionega ción niños no desa rollado organizacionea paaso país periodo perjudicial planeta

políticos pánico reducción respaldo responsabilidad salvar sociedad soluciones tiempovoto

Fuente: Elaboración propia en Atlas.ti (2020). 
Se muestran ilustradas las unidades de análisis encontradas en el discurso, siendo las palabras con mayor jerarquía visual aquellas con mayor recurrencia, definidas por la saturación de color y el tamaño de letra.

Gráfico 1: Cantidad de publicaciones mensuales con la palabra "cambio climático" anteriores al discurso de José Mujíca

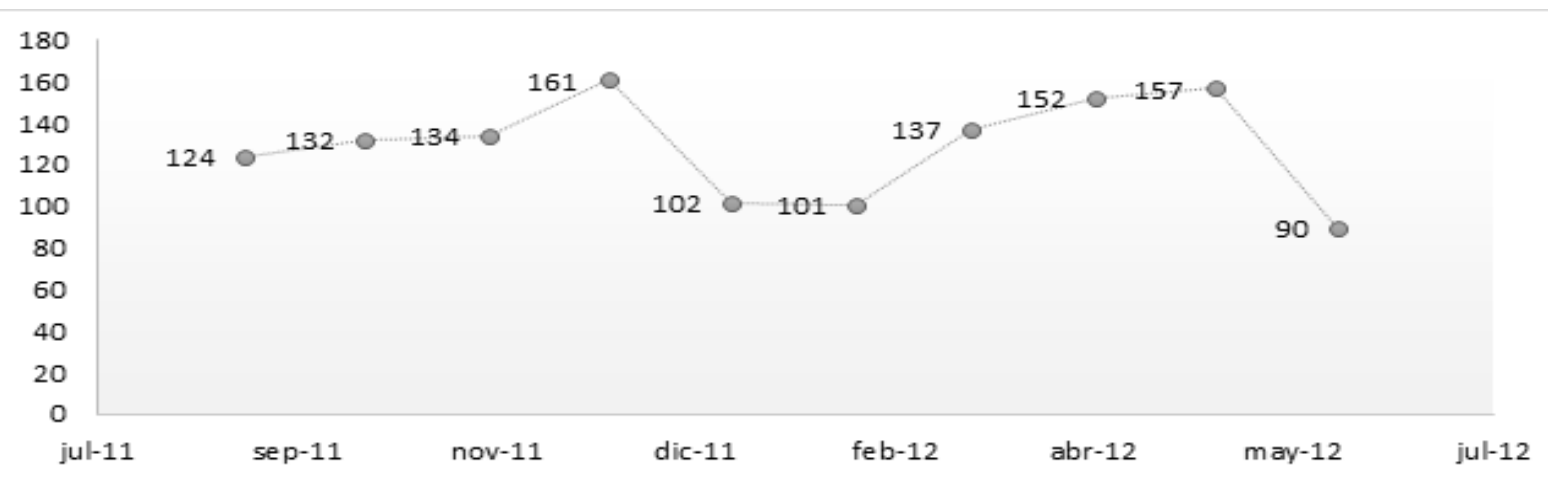

Fuente: Elaboración propia (2020).

El gráfico muestra el número de publicaciones mensuales encontrados en ProQuest de acuerdo al filtro 1.1 de las categorizaciones temporales del análisis de resultados, siendo los 10 meses anteriores al discurso de José Mujica, esto permite observar el comportamiento de las publicaciones y percibir las transformaciones posteriores.

Gráfico 2: Cantidad de publicaciones mensuales con la palabra "cambio climático" posteriores al discurso de José Mujíca.

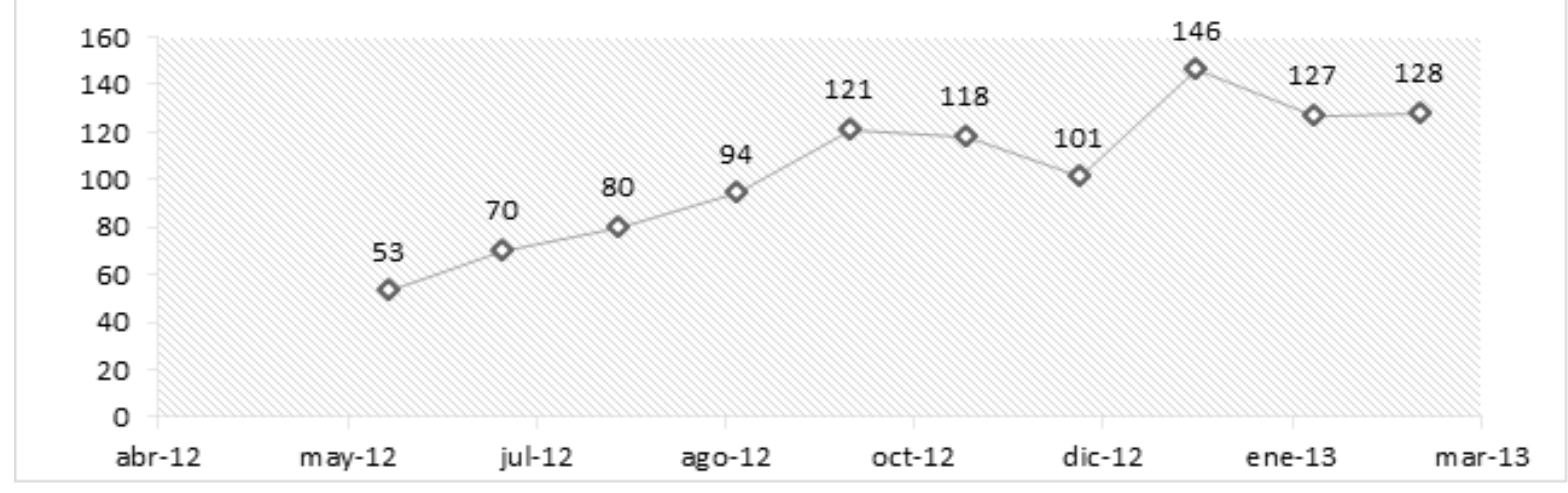

Fuente: Elaboración propia (2020). 
El gráfico muestra el número de publicaciones mensuales encontradas en ProQuest de acuerdo al filtro 1.2 de las categorizaciones temporales del análisis de resultados, siendo los 10 meses posteriores al discurso de José Mujica, esto permite observar el comportamiento de las publicaciones y el efecto sobre la prensa a causa del discurso.

Gráfico 3: Cantidad de publicaciones mensuales con la palabra "cambio climático" anteriores al discurso de Greta Thunberg.

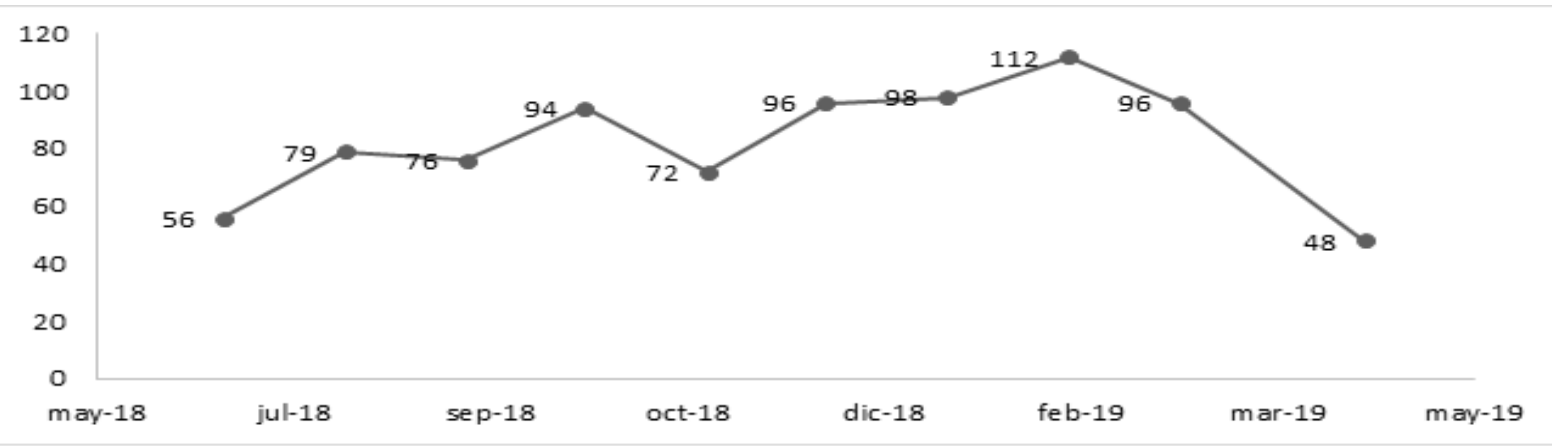

Fuente: Elaboración propia (2020).

El gráfico muestra el número de publicaciones mensuales encontradas en ProQuest de acuerdo al filtro 2.1 de las categorizaciones temporales del análisis de resultados, siendo los 10 meses anteriores al discurso de Greta Thunberg, esto permite observar el comportamiento de las publicaciones antes del discurso y mostrar la viscitud.

Gráfico 4: Cantidad de publicaciones mensuales con la palabra "cambio climático" anteriores al discurso de Greta Thunberg.

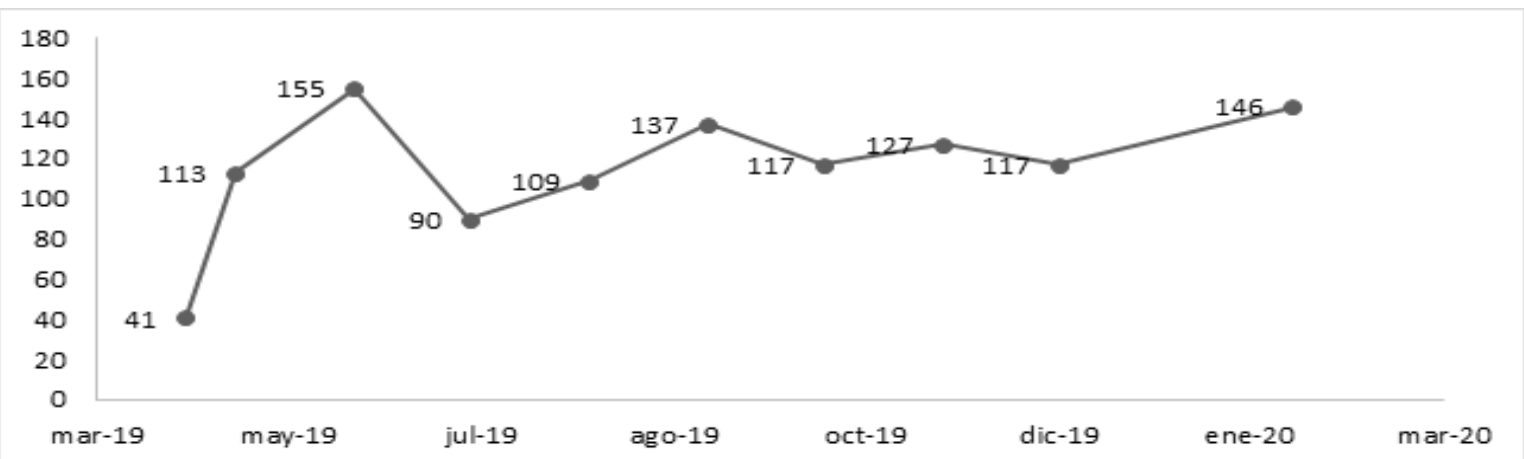

Fuente: Elaboración propia (2020). 
El gráfico muestra el número de publicaciones mensuales encontrados en ProQuest de acuerdo al filtro 2.2 de las categorizaciones temporales del análisis de resultados, siendo los 10 meses posteriores al discurso de Greta Thunberg, esto permite observar el comportamiento de las publicaciones, la evolución y comparar con la gráfica anterior.

Gráfico 5: Filtro 3. Cantidad de publicaciones anuales con la palabra "cambio climático" entre la emisión discursos.

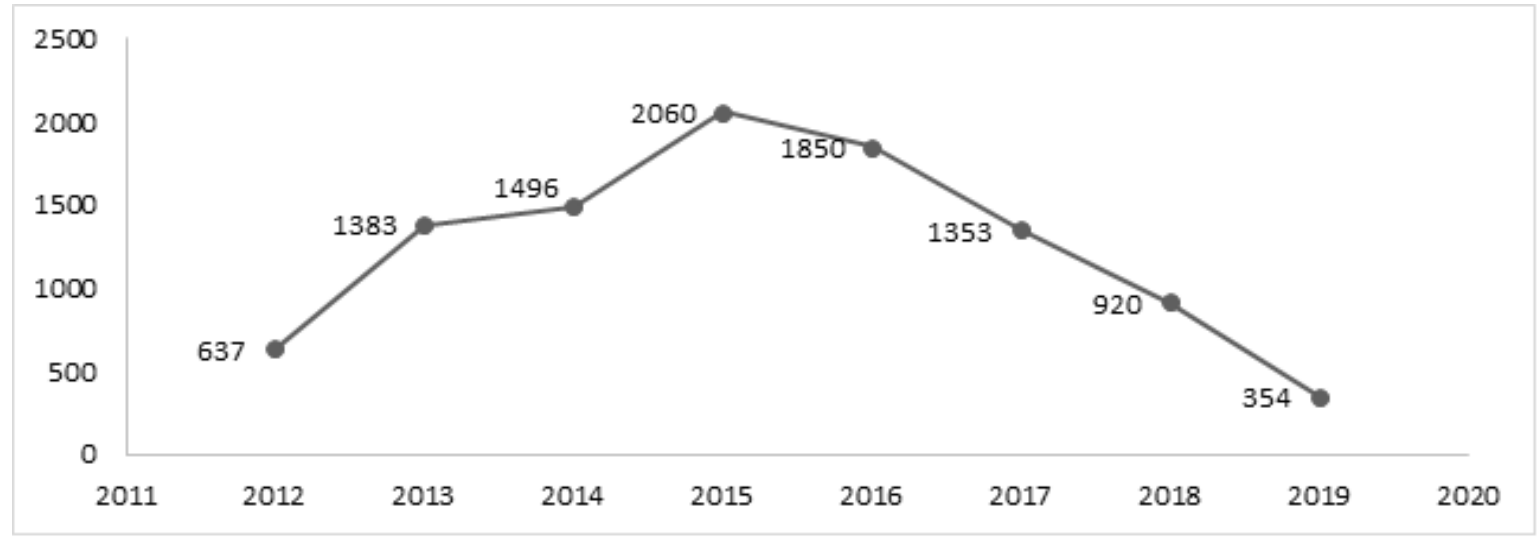

Fuente: Elaboración propia (2020).

El gráfico muestra el número de publicaciones mensuales encontradas en ProQuest de acuerdo al filtro 3 de las categorizaciones temporales del análisis de resultados, observando de manera general el comportamiento de las publicaciones, y la frecuencia de las mismas. 
86

Tabla 1: Conceptos encontrados en las publicaciones, adicionales a "cambio climático".

\begin{tabular}{|c|c|c|}
\hline No. & Tema & Cuenta \\
\hline 1 & Cambio climático & 2,136 \\
\hline 2 & Emisiones & 663 \\
\hline 3 & presidentes & 631 \\
\hline 4 & calentamiento global & 533 \\
\hline 5 & protección del medio ambiente & 528 \\
\hline 6 & carbono & 492 \\
\hline 7 & campañas políticas & 446 \\
\hline 8 & estudios & 445 \\
\hline 9 & gas natural & 285 \\
\hline 10 & política de energía & 284 \\
\hline 11 & plan industrial de emisiones & 273 \\
\hline 12 & elecciones presidenciales & 250 \\
\hline 13 & políticas & 249 \\
\hline 14 & industria petrolera & 231 \\
\hline 15 & políticas medioambientales & 228 \\
\hline 16 & industria energética & 219 \\
\hline 17 & partidos políticos & 219 \\
\hline 18 & cuentas & 213 \\
\hline 19 & efecto invernadero & 202 \\
\hline 20 & nominaciones & 195 \\
\hline 21 & combustibles fósiles & 187 \\
\hline 22 & investigadores & 177 \\
\hline 23 & candidatos & 170 \\
\hline 24 & liderazgo & 167 \\
\hline 25 & primer ministro & 159 \\
\hline 26 & fuentes de energía alternativa & 142 \\
\hline 27 & industria automotriz & 140 \\
\hline 28 & carbón & 135 \\
\hline 29 & centrales eléctricas de carbón & 132 \\
\hline 30 & tuberías & 130 \\
\hline 31 & nivel del mar & 128 \\
\hline 32 & gases de efecto invernadero & 127 \\
\hline 33 & escuelas \& universidades & 121 \\
\hline 34 & sequía & 120 \\
\hline
\end{tabular}


35 acuerdos

36 dióxido de carobono

37 recesión

38 ciudades

39 crecimiento económico

40 libros

41 relaciones internacionales 109

42 inversiones 104

43 producto interno bruto (PIB) 102

44 investigación 102

45 editoriales 99

46 leyes y regulaciones de armas de fuego 98

47 redes sociales 97

48 impuestos del medioambiente 96

49 inundaciones 96

50 ciencia 95

51 primaries \& asambleas 94

52 hielo 92

53 gobernadores 89

54 especies en peligro de extinción y extintas 87

55 acuerdo de París $\quad 87$

56 lluvia 87

57 servicios públicos eléctricos 86

58 litigación 86

59 científicos 86

60 políticas extranjeras 85

61 clima 85

62 legisladores $\quad 84$

63 tecnología limpia 83

64 servicios diplomáticos y consulares 83

65 tasas de impuestos $\quad 80$

66 presupuestos 81

67 comida 81

68 incendios forestales y de maleza $\quad 81$

69 contaminación del aire 80

70 financiación 79

71 seguridad nacional 79

$\begin{array}{ll}72 & \text { utilidades del gas natural }\end{array}$ 
73 emisiones estandares 78

74 cumbre de conferencias económicas $\quad 77$

75 infraestructura 76

76 lider político $\quad 76$

77 agricultura $\quad 74$

78 tierra 74

79 inmigración $\quad 74$

80 regulación 74

81 precios 73

82 vehículos eléctricos 71

83 coperación $\quad 69$

84 comercio de emisiones 69

85 decisiones de la corte federal 69

86 industria de carbón $\quad 68$

87 eficiencia energética $\quad 68$

88 estudiantes $\quad 68$

89 subsidios $\quad 67$

90 intereses 66

91 política de inmigración $\quad 65$

92 aliens $\quad 64$

93 ecosistemas $\quad 64$

94 bancos centrales 63

95 costos 63

96 hurracanes $\quad 62$

97 legislación $\quad 62$

98 abogados generales $\quad 61$

99 medioambientalistas $\quad 61$

Fuente: Elaboración propia (2020).

En esta tabla podemos observar los temas más localizados y recurrentes, de manera jerarquica, en las publicaciones encontradas de ProQuest en el periodo comprendido entre los dos discursos (7 años) del Filtro temporal 3, lo cual muestra la relación que tienen con "cambio climático", esto favorece la comprensión del problema de forma general, y en el contraste con las unidades de análisis del discurso de José Mujíca, ya que por la temporalidad solo permite su contemplación, porque no abarca un periódo posterior al discurso de Greta Thunberg que permita 
el análisis completo.

\section{5.- CONCLUSIONES}

Una cantidad indefinida de factores intervienen en los procesos de investigación y análisis, por lo que proponer una respuesta única e incuestionable en el ámbito social no es factible. Por ejemplo, la base de datos muestra un sesgo hacia los medios estadounidenses por que la misma base proviene de Estados Unidos de América, condicionante que se debe tomar en cuenta. De acuerdo con los resultados obtenidos, se puede inferir que ciertos discursos sí tienen un impacto relevante en la creación de notas informativas, lo que sí se puede concluir es que los discursos no son hechos aislados, tal vez los eventos seleccionados no tuvieron una gran repercusión en los medios, no obstante, si lograron posicionar la problemática medio ambiental en el interés público, preparando a la audiencia, y a los medios, para los discursos posteriores, que la información o acciones futuras enfocadas en la resolución del cambio climático sí logren el alcance que se requiere para generar un cambio.

Demostrando de esta forma que los discursos se convierten en hechos noticiosos porque los medios producen el contenido que la gente consume por ello es importante entender que el aumento de notas informativas no fue iniciativa exclusiva de los medios, al contrario, el interés de la población aumento en paralelo y con ello se han incorporado adeptos y activistas en el tema. Con esto se puede decir que los discursos realmente cumplieron su objetivo, ya no son figuras publicas aisladas las que piden un cambio y toma de acciones, si no un grupo que aumenta día con día, de personas preocupadas y ocupadas por un mejor futuro.

Demostrando de igual forma cómo la teoría de comunicación en dos pasos de Paul Lazarsfeld sigue estando vigente porque, al final, gracias a las noticias publicadas en los medios, en específico el periódico, surgirán más adeptos al tema, y más personas se interesarán en resolver la problemática. Esto a nivel profesional puede tener mayor impacto sobre las personas enfocadas en crear contenidos que sobre los mismos activistas medioambientales, ya que la repercusión puede aumentar al 
mostrar información en un medio masivo a comparación del público segmentado de un activista, demostrando el poder tan valioso con el que cuentan los medios al tener un impacto global.

En el caso del análisis de resultados, el lenguaje demuestra ser un reflejo de la realidad, al menos en el discurso, puesto que se pudo posicionar a partir del análisis comparativo y a partir de la comprensión del contexto del que provienen, la situación que se vive en los distintos momentos temporales en los que se crearon los discursos.

El estudio y análisis de resultados que se generó al final, fungió como radiografía general de la situación, porque de este punto pueden surgir múltiples investigaciones que tengan un objeto de análisis más específico y delimitado, a causa de que existen diversos aspectos a considerar dentro de este artículo, como por ejemplo el matiz de las notas publicadas, porque el hecho de que contenga el concepto cambio climático no implica que sea una postura a favor, sino todo lo contrario; otro ejemplo sería el análisis por región; pero sobre todo una mayor detención en el contenido de las notas, fragmentar y analizar por completo la postura de los periódicos o medios más importantes y a partir de ello proponer cambios y soluciones.

Sin embargo, la conclusión más importante que surge es la complejidad del problema medioambiental, que a pesar de lo que múltiples especialistas demuestren, no solo los científicos y políticos pueden generar un cambio, todos los ámbitos pueden y deben crear acciones al respecto, demostrando con este estudio la importancia del rol de la comunicación y las ventanas de oportunidad que se deben trabajar porque al final, el cambio está en todos.

\section{REFERENCIAS}

Arias, M. y Rosales, S. (2019). Educación ambiental y comunicación del Cambio Climático. Revista Mexicana de Investigación Educativa, 24 (80), 247-269. 
Audi, R. (2014). Diccionario Akal de Filosofía, 2da edición. Madrid (España): Akal ediciones.

Belam, M. (septiembre 26, 2019). Greta Thunberg: teenager on a global mission to 'make a difference'(The Guardian). https://www.theguardian.com/ environment/2019/sep/26/greta-thunberg-teenager-on-a-global-mission-tomake-a-difference

Cárdenas, M. (2010). Calentamiento global y cambio climático: El tratamiento del problema en los medios de comunicación mexicanos 2008. Tesis Doctoral. Universidad de Zaragoza, Zaragoza, España.

Dahdah-Antar, S. (2019). El imperio retórico: Auge y Caída. Revista Política, Globalidad y Ciudadania, 5(9), 106 -123. http://revpoliticas.uanl.mx/index. php/RPGyC/article/view/111

El País. (agosto 14, 2018). Mujíca y su largo viaje en la política. (El País). https:// elpais.com/elpais/2018/08/13/album/1534153764_603271.html\#foto_gal_1

Foucault, M. (1987). El orden del discurso. Barcelona, España: Tusquets.

Hernández, J. y García, M. (1994). Historia breve de la retórica. (19 ed., p. 210212). Estudios de Literatura.

IAB México. (marzo 2017). Estudio de Consumo de Medios y Dispositivos entre Internautas Mexicanos. IAB México, Televisa Digital \& Kantar Millwardbown, México, 9.

Knight, F. (1850). The Fourth Estate: Contributions Towards a History of Newspapers, and of the Liberty of the Press. Australia: Wentworth Press.

Lazarsfeld, P., Berelson, P., y Gaudet, H. (1944). The people's choice: how the voter makes up his mind in a presidential campaign. Columbia: University Press, p. 151.

Lazarsfeld, P. (2012). The Rise of Limited-Effects Theory en Baran, S. y Davis, D (Ed.). Mass Comunication Theory. Foundations, Fermet, and Future. ( $6^{\mathrm{o}} \mathrm{ed}$, p. 142-145). Wadsworth: Cengage Learning. 
Oceana. (S.f.). Gases de efecto invernadero. Washington, D.C, EU.: EU Funding Support. https://eu.oceana.org/es/node/46897

Organización de las Naciones Unidas. (S.f.). Introducción al cambio climático. https://unccelearn.org/course/view.php?id=13\&page=overview

Organización de las Naciones Unidas. (2012). El futuro que queremos. Río+20 Conferencia de las Naciones Unidas sobre el Desarrollo Sostenible. Río de Janeiro: Brasil.

Phillips, N. y Hardy, C. (2002). Discourse Analysis: Investigating processes of Social Construction. Sage University Paper Series on Qualitative Research Methods. (Vol. 50). Sage.

Rico, A. (2004). Análisis Sociolingüístico de los Discursos Ambientales sobre la Amazonía. Gestión y Ambiente. 7(2), 5-14.

Ricoeur, P. (1995). Teoría de la interpretación: Discurso y excedente de sentido. México: Siglo XXI.

Van Djik, T. (1978). La ciencia del texto. España: Paidós.

Van Djik, T. (2012). Discurso y contexto. México: Editorial Gedisa.

Velázquez De Castro, F. (agosto 2012). La crisis ambiental y la deuda ecológica. (Revista Crítica). http://www.revista-critica.com/la-revista/monografico/ analisis/283-la-crisis-ambiental-y-la-deuda-ecologica 